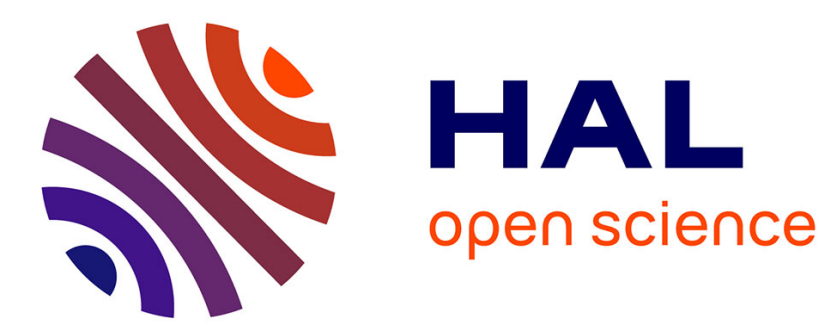

\title{
Politiques d'insertion et citoyenneté sociale des jeunes
} Lea Lima

\section{To cite this version:}

Lea Lima. Politiques d'insertion et citoyenneté sociale des jeunes. Becquet Valérie, Loncle Patricia, Van de Velde Cécile (dir.). Politiques de jeunesse: le grand malentendu, Champ social, pp. 126-137, 2012, Questions de société, 978-2-35371-290-8. halshs-00833397

\section{HAL Id: halshs-00833397 https://shs.hal.science/halshs-00833397}

Submitted on 12 Jun 2013

HAL is a multi-disciplinary open access archive for the deposit and dissemination of scientific research documents, whether they are published or not. The documents may come from teaching and research institutions in France or abroad, or from public or private research centers.
L'archive ouverte pluridisciplinaire HAL, est destinée au dépôt et à la diffusion de documents scientifiques de niveau recherche, publiés ou non, émanant des établissements d'enseignement et de recherche français ou étrangers, des laboratoires publics ou privés. 


\section{Politiques d'insertion et citoyenneté sociale des jeunes}

Léa Lima ${ }^{1}$

L'importance des politiques d'insertion des jeunes et leur rôle structurant dans les politiques de jeunesse occultent encore de nos jours la question des droits sociaux pour les jeunes. En se penchant sur le sort réservé aux séniors, de nombreux chercheurs ${ }^{2}$ ont mis en lumière la dimension «âgisée » ${ }^{3}$ des politiques sociales. C'est moins le cas lorsqu'on aborde les politiques sociales de jeunesse. Les politiques publiques envers les jeunes sont auscultées, évaluées sous l'angle de leur efficacité mais rarement sous l'angle de l'accès aux droits. C'est que les jeunes en France sont des objets d'intervention sociale avant d'être des sujets de droit. Pourtant les politiques d'insertion drainent un ensemble de prestations sociales qu'elles soient sous forme de services ou sous forme d'allocations. Parce qu'elle est un impensé des politiques publiques, la citoyenneté sociale des jeunes se dessine en creux. Elle est mitée par des règles d'âge spécifiques à chaque secteur de l'action sociale, créant des plages d'incohérence dont les acteurs locaux doivent se débrouiller. A ces règles d'âge, figées dans le droit, s'ajoutent des normes d'âge (Bessin, 1994) structurantes des politiques d'insertion, c'est-à-dire des attentes sociales formulées par rapport à chaque classe d'âge.

\section{La production du maquis des règles d'âge dans la législation sociale}

Les politiques sociales de jeunesse ont une histoire longue qui produit une forme de pesanteur sur les nouvelles initiatives car elles sont intégrées comme une donnée, une contrainte pour l'action publique future. Plus le temps passe, plus les mesures s'amoncellent et se chevauchent, plus il devient difficile de remettre à plat l'ensemble du système tant l'interdépendance entre des pans des politiques sociales est forte.

\section{Les mobiles de la discrimination sociale selon l'âge}

Les mobiles avancés pour réserver un traitement spécifique aux jeunes sont maintenant bien connus et sont relativement stables dans le temps. Les mêmes arguments qui ont présidé à l'éviction des jeunes du RMI en 1988 ont été utilisés dans le débat sur le RSA. Il est prétexté le risque de trappe à inactivité ou de désincitation au travail provoqué par la perspective d'un revenu sans contrepartie. C'est une variante de la crainte de la désincitation à la formation qui structure les politiques de l'emploi des jeunes. Lors du débat sur le RSA, Pierre Méhaignerie déclare par exemple en commission à l'Assemblée que «beaucoup d'entre eux [les jeunes] commencent leur vie professionnelle par l'intérim. Pour certains, cette activité dure quelques mois puis ils passent au chômage afin de disposer d'une liberté de vie pour voyager ou

\footnotetext{
$1 \quad$ Léa Lima est maître de conférence au Conservatoire National des Arts et Métiers, sociologue au LISE. 2 Voir l'ouvrage d'Anne-Marie Guillemard (2003) pour un état de l'art sur la question.

3 Nous employons ce terme comme équivalent dans le domaine de l'âge de l'adjectif « genré » pour les sexes. Il désigne le caractère socialement construit des différences d'âge.
} 
compléter leur formation, financée en partie par l'assurance-chômage. Ainsi, $40 \%$ des jeunes ne veulent pas passer en contrat à durée indéterminée. C'est parfois, dans les premières années de travail, un choix de mode de vie, même s'il faut relativiser ce constat effectué dans un bassin d'emploi où le taux de chômage est de $4 \% »{ }^{4}$. L'âge biologique est alors significatif d'un âge psychique, celui de l'immaturité, de l'inconscience ou de la légèreté qui accentuerait chez les jeunes la préférence pour les loisirs. C'est cette même immaturité psychique qui sous-tend l'argument moral selon lequel « on ne peut offrir l'assistanat comme perspective aux jeunes de notre pays $»^{5}$. Ce type d'assertion sous-entend en effet que cette perspective serait plus acceptable pour les personnes de plus de 25 ans car ils ne pourront la considérer comme la norme statutaire.

Un second argument concerne l'âge social lié à l'accès aux droits, qui permet d'invoquer l'obligation alimentaire ou la nécessité d'une solidarité familiale accrue ${ }^{6}$. L'âge biologique est alors un repère statutaire qui fixe un ensemble de droits et d'obligations spécifiques : ceux de l'enfance, de la conjugalité ou de la parentalité. L'ouverture du RMI aux jeunes de moins de 25 ans parents d'enfants nés ou à naître prenait d'ailleurs acte de la norme sociale moderne implicite de la solidarité intergénérationnelle qui veut que l'on cesse quelque peu d'être l'enfant débiteur de ses parents à partir du moment où l'on devient père ou mère soi-même. En mixant les deux logiques (âge psychique, âge social) on aboutit à la décision d'ouvrir des droits au RSA pour les jeunes de moins de 25 ans présentant des signaux forts d'intégration dans le monde du travail (deux ans d'activité salariée sur une période de trois ans). Leur ancienneté sur le marché de l'emploi marque leur attachement (psychique) au travail et diminue, du point de vue des décideurs, les risques de développement d'une "culture de l'assistanat »; par ailleurs elle les rapproche de la norme d'autonomie qui rend le retour dans le giron de la solidarité familiale plus difficile.

Enfin un dernier argument, plus pragmatique, invoque les solutions alternatives existantes. Il est vrai que le champ des politiques d'insertion des jeunes entre 16 et 25 ans s'est largement institutionnalisé (Lima, 2008) et qu'un individu bien accompagné peut circuler jusqu'à ses 25 ans dans le maquis des mesures d'insertion et des aides sociales locales qui lui apporteront à chaque fois un subside: Fonds d'aide aux jeunes, CIVIS, Contrat jeune majeur, Contrat d'Accès à l'Autonomie, formations professionnelles subventionnées par le Conseil Régional, contrats aidés des secteurs marchands et non marchands. Cet argument rationalise d'une certaine manière le sentier de dépendance des discriminations selon l'âge qui fait que les choix passés en termes de définition des âges sociaux déterminent fortement les choix présents.

\section{Le sentier de dépendance des discriminations sociales selon l'âge}

\footnotetext{
$4 \quad$ Rapport fait au nom de la commission des affaires culturelles, familiales et sociales sur le projet de loi ( $\left.n^{\circ} 1100\right)$ généralisant le revenu de solidarité active et réformant les politiques d'insertion par M. Marc-Philippe DAUBRESSE, Assemblée Nationale, $N^{\circ} 1113$.

$5 \quad$ Roselyne Bachelot, réponse à la question n²887, Assemblée nationale, le 26/01/2011.

6 Précisons que rien ne permet dans le code civil de poser une limite d'âge à l'obligation alimentaire.
} 
La référence à l'âge biologique est omniprésente dans la tranche 16-25 ans. Entre 16 ans, âge de la fin de la scolarité obligatoire et 25 ans, âge de la majorité fiscale pour les étudiants, deux bornes apparaissent comme structurantes : 18 et 21 ans $^{7}$. Ces deux bornes sont empruntées à la majorité civile. Les législations sociales introduites avant 1974, date à laquelle Valéry Giscard d'Estaing décide d'abaisser l'âge de la majorité, ont conservé la trace des 21 ans. Afin de ne pas déstabiliser le travail social développé par les services de protection de l'enfance qui entouraient l'enfant en danger (et/ou considéré comme dangereux) jusqu'à sa majorité, le gouvernement de Jacques Chirac décide en 1975 de maintenir une protection pour les jeunes sortants de l'Aide Sociale à l'Enfance (ASE) entre 18 et 21 ans $^{8}$. C'est ainsi qu'aujourd'hui encore le contrat jeune majeur de l'ASE offre entre 18 et 21 ans une allocation qui concurrence de manière avantageuse la bourse CIVIS ouverte aux jeunes entre 18 et 25 ans ${ }^{9}$.

Les limites d'âge trahissent le caractère social des politiques d'insertion. C'est le cas des barèmes de rémunération des stagiaires de la formation professionnelle (loi de 1971) qui encore aujourd'hui sont échelonnés en fonction de l'âge (16-18 ans ; 18-21 ans ; 21-25 ans, 25 ans et plus). La recherche de cohérence entre les mesures d'insertion ne s'est pas faite par la réduction des strates d'âge dans les anciennes législations mais par la reproduction de l'ensemble des sous-catégories d'âge dans les nouvelles règlementations. Ainsi les contrats en alternance massivement introduits dans la première moitié de la décennie 1980 conservent-ils les bornes d'âge des 21 ans et des 25 ans (mais pas celle des 18 ans). Les rémunérations des contrats d'apprentissage ainsi que des contrats de professionnalisation s'échelonnent en fonction du niveau de qualification et de l'avancement dans la formation mais aussi en fonction de l'âge biologique.

Le seuil des 25 ans utilisé dans le RMI puis le RSA découle de la définition de la notion d'enfant à charge qui stipule que l'enfant peut être déclaré par ses parents comme à charge jusqu'à ses 25 ans s'il est étudiant (21 ans sinon). Le Fonds d'aide aux jeunes créé en 1989 comme un ersatz de revenu minimum a naturellement couvert cet intervalle de temps entre 18 et 25 ans pendant lequel la majorité civile ne coïncide pas avec la majorité sociale. Ces emboîtements de législation ont créé un véritable maquis de droits devenu de plus en plus illisible pour les principaux intéressés. Parce que certaines de ces dérogations à la majorité civile donne des avantages et aménagent des protections, les modulations selon l'âge ne sont que rarement dénoncées comme des discriminations d’âge (Poli, Lefrançois, Cradec, 2009).

\section{Quelles spécificités des politiques sociales de jeunesse?}

La persistance d'une politique sociale spécifique aux jeunes (appelons-la «politique d'insertion des jeunes »), autrement dit la persistance de catégories d'âge dans les politiques

\footnotetext{
$7 \quad$ Il faudrait y ajouter celle de 20 ans pour la définition d'enfant ayant-droit dans le code de la sécurité sociale (si l'enfant est étudiant) et d'enfant à charge au sens de la caisse d'allocations familiales (si toutefois il perçoit une rémunération inférieure à $55 \%$ du SMIC ).

9 Décret $\mathrm{n}^{\circ} 75-1118$ du 2 décembre 1975. montant de l'allocation mensuelle dans le cadre du contrat jeune majeur est fixé par les Conseils Généraux mais il est souvent indexé sur le montant du RSA socle.
} 
sociales qui exclue les jeunes majeurs d'un certain nombre de droits est d'autant plus incompréhensible que les principes qui en guident l'action rejoignent largement les politiques sociales pour les « adultes ».

Le référentiel de l'accompagnement y est prédominant et n'a fait que se renforcer depuis 30 ans. On peut même penser que les politiques d'insertion des jeunes ont ouvert la voie en la matière. Les compétences des professionnels de l'insertion sont donc peu ou prou les mêmes dans les missions locales et les services d'insertion des conseils généraux (qui pilotent le RSA) : ils doivent connaître l'offre de services de formation, d'emploi, et les services sociaux sur leur territoire de manière à en faire bénéficier " en temps voulu » les bénéficiaires dont ils ont le suivi. On leur demande de plus en plus de pratiquer une forme de " case management» consistant à coordonner dans le temps des parcours individuels, des interventions réalisées par d'autres, la fonction d'écoute et de conseil devenant de plus en plus secondaire. Parallèlement on a vu se généraliser, ici plutôt des adultes vers les jeunes, la notion de contractualisation et de responsabilisation des bénéficiaires. Le contrat d'insertion dans le RMI-RSA a été largement discuté mais il est tout aussi présent dans les politiques d'insertion des jeunes : il n'est pas un programme d'accompagnement (TRACE puis CIVIS, FAJ) qui ne présente un contrat d'engagement à signer par la personne. Le discours sur la flexicurité et l'éducation tout au long de la vie a affaibli en retour la spécificité fonctionnelle des politiques d'insertion des jeunes. Dans les années 1980, ces dernières pouvaient encore être définies autour de la notion de qualification professionnelle. Si les transitions et les requalifications des individus se multiplient au cours de la vie active, la segmentation par catégorie d'âge devient moins pertinente. La stratification par âge des mesures ne tient donc que par la discrimination dans l'accès aux droits sociaux ouvrant le versement d'un revenu.

\section{La police des âges en action : les normes d'âge dans le Fonds d'aide aux jeunes (FAJ)}

Les observations des délibérations au sein de commissions d'attribution des fonds départementaux d'aide aux jeunes montrent les normes biographiques en action. Le FAJ est un dispositif obligatoire de l'action sociale mis en œuvre par les Conseils Généraux. Il apporte une aide financière ponctuelle aux jeunes en difficultés entre 18 et 25 ans pour la subsistance ou la réalisation d'un projet d'insertion. L'exposé des dossiers de demande d'aide convoque en permanence des repères d'âge, qu'ils soient imposés par les dispositifs d'insertion ou qu'ils soient liés à des normes d'employabilité.

\section{L'effet des bornes d'âge sur l'accompagnement des jeunes}

Soulignons tout d'abord que l'âge (et non la date de naissance) forme une des principales informations que les décideurs possèdent systématiquement sur le demandeur. L'exposé oral de la situation des individus commence donc invariablement par l'énoncé du sexe et de l'âge. Alors que l'on ne connaît pas forcément d'emblée leur situation matrimoniale. L'action des professionnels de l'insertion des jeunes est soumise aux contraintes de la "police des âges » (Percheron, 1991). Ils assument alors une fonction de "tuilage» des dispositifs dans les trajectoires d'insertion des jeunes. L'âge de 21 ans est un seuil marquant des trajectoires des jeunes et un repère pour les professionnels. Il marque la fin de la prise en charge pour un 
certain nombre de jeunes en grande difficulté qui bénéficiaient de la couverture de l'ASE. Outre le pécule auquel ils avaient droit dans le cadre du contrat jeune majeur, c'est aussi une offre de services liés à leur statut qui se referme.

\section{Bornes d'âge et ruptures d'accompagnement : le cas d'Eve}

Eve va sur ses 21 ans et arrive au terme de son suivi en Contrat Jeune Majeur (CJM) par le service de l'Aide Sociale à l'Enfance (ASE) du fait de la limite d'âge ; elle est connue depuis 15 ans de ces mêmes services et se retrouve sans hébergement. Elle dort depuis 9 jours à l'hôtel. Une technicienne du Conseil Général qui préside le Comité Local d'Attribution se pose ici en garante de la cohérence de l'action sociale sur le territoire : [agacée] «Moi ce qui m'épate toujours, c'est que c'est une jeune qui est en Contrat Jeune Majeur, on sait pertinemment que les 21 ans arrivent, qu'est-ce qui a été travaillé sachant que les 21 ans arrivent ? Ça, moi, je suis toujours épatée ! ». Le comité, composé de V., pour le Conseil Général, une représentante permanente de la $\mathrm{CAF}$, une conseillère de la mission locale, et une chef de service de la Maison Départementale des Solidarités (soit le guichet unique des services sociaux du Conseil Général), est l'occasion de refaire l'histoire de la prise en charge de la jeune femme et de pointer tous les dysfonctionnements, ceux de l'ASE en l'occurrence (non représentée à la commission). Cet exemple est assez révélateur des effets relativement délétères des bornes d'âge dans les politiques sociales. L'anniversaire des 21 ans provoque non seulement l'arrêt brutal du versement de l'allocation liée au contrat mais aussi une restriction dans l'offre de places en foyers d'hébergement. Il déclenche de plus un changement de référent : l'assistante sociale de l'ASE passe le relais à l'assistante sociale de secteur (Conseil Général). Les intervenants en commission mettent en regard le temps long de l'accompagnement («15 ans d'accompagnement pour en arriver là ») et la brutalité de l'évènement. L'anniversaire des 21 ans est vécu comme une rupture biographique importante par Eve elle-même qui exprime ainsi à l'assistante sociale de secteur son désarroi : «j'ai l'impression qu'on m'a monté la tête, on m'a mis dans un truc, et là, je me retrouve toute seule face à une merde, je n'ai personne à côté de moi ».

Les départements en charge de l'action sociale introduisent leurs propres limites d'âge : l'Essonne par exemple a mis en place pour les 16-19 ans, une «carte jeune » sur le principe du chéquier à dépenser auprès de partenaires. Aux bornes d'âge légales qui structurent de fait l'intervention sociale, il faut ajouter les repères de nature plus conventionnelle qui sont aussi des ajustements de sous-systèmes de l'action sociale. Dans certains départements, la barrière d'âge de 21 ans a été reprise par la préfecture en charge de la gestion des places d'hébergement d'urgence sociale dit du « $115 »$ (du numéro des urgences sociales qui opère la veille sur les places vacantes dans les structures du département). En toute illégalité, le 115 refuse ainsi de traiter les demandes d'hébergement des jeunes majeurs de moins de 21 ans en les renvoyant vers le conseil général. 
Dans les délibérations du FAJ, les acteurs font régulièrement référence à l'âge du demandeur, surtout pour les âges " extrêmes ", c'est-à-dire ceux qui sont proches des bornes inférieure et supérieure, 18 et 25 ans $^{10}$. Un jeune de « 18 ans et demi » (comme le précise la présentation orale du dossier) est «tout jeune », un autre de 19 ans « tout jeunot». Souvent ce jeune âge est invoqué pour justifier du report du projet de formation ambitieux et onéreux. Le premier a arrêté son cursus en $1^{\text {ère }}$ année de CAP d'industrie car la filière ne lui plaisait pas. Il a enchaîné un certain nombre de missions d'intérim au cours desquelles, selon son conseiller, il " a découvert le métier de manutentionnaire et le déroulement du travail en entrepôt lui a plu ». Il fait une demande pour le CACES, un certificat d'aptitude permettant d'exercer la fonction de cariste ${ }^{11}$. Les membres de la commission s'interrogent sur la pertinence d'un CACES « sec » qui ne soit pas accompagné d'une formation qualifiante, ce qui nécessiterait d'attendre que des places s'ouvrent dans les organismes de formation. Pour la commission, « c'est un jeune qui peut attendre », vu le parcours et l'âge ; "s'il se bouge un peu, il peut travailler un peu en attendant un prochain recrutement ».

A partir d'un certain seuil qui n'est pas totalement figé, l'âge devient au contraire un handicap dans l'accès aux formations longues dans la mesure où l'on considère alors que l'accès rapide à l'emploi est une priorité. Un jeune de 22 ans ou plus qui n'a pas accumulé un certain nombre d'expériences risque d'être stigmatisé car soupçonné de ne pas faire tout son possible pour travailler. Pour les jeunes de bas niveau de qualification dont il est question ici, la fenêtre de la reprise d'études est donc relativement étroite : pas trop précoce pour faire la preuve que l'on n'a pas peur du travail et pour se constituer une petite épargne à réinvestir dans le projet de formation, pas trop retardée pour pouvoir bénéficier de l'aide financière de ses parents et ne pas être sanctionné sur le marché du travail.

\section{Conclusion}

Chaque élection présidentielle suscite l'espoir d'une mise à plat des politiques sociales de jeunesse. Et à chaque fois c'est la frilosité qui l'emporte sur fond d'organisations de jeunesse atones. Les efforts des gouvernements successifs se concentrent sur l'accès à l'emploi jugeant à juste titre que c'est une préoccupation majeure des Français. Pourtant les possibilités d'innovation en matière de politiques d'insertion des jeunes sont largement réduites si l'on considère comme intouchables deux fondamentaux des politiques de jeunesse : une politique d'éducation et de formation monochrone sur le modèle de l'étudiant à plein temps et sans interruption d'une part, une politique sociale discriminatoire en termes de droits d'autre part. Une véritable politique d'éducation tout au long de la vie (qui ferait notamment tomber les limites d'âge dans l'octroi de toutes les bourses de l'Education nationale et de l'enseignement supérieur) permettrait de desserrer l'étau autour des trajectoires accidentées. Une politique sociale moins « âgisée » pourrait être plus attentive aux singularités. C'est une condition pour la mise en œuvre de politiques de situations et non de statuts.

\footnotetext{
10 On a là un exemple relativement classique de biais de perception liés à la fenêtre d'observation des parcours : ceux qui sont « vieux» dans un dispositif le seraient moins si on les comparait à la population générale. Ceux qui sont « vieux » dans le FAJ deviennent « jeunes » en entrant dans le RSA.

Les caristes travaillent comme manutentionnaires dans les entrepôts et manipulent des engins pour porter les palettes de produits.
} 


\section{Bibliographie}

M. BESSIN, « La police des âges entre rigidité et flexibilité temporelles. Première partie : la chronologisation du cours de vie », Temporalistes, $\mathrm{n}^{\circ} 27$, septembre, 1994, pp. 8-13.

A.-M. Guillemard, L'âge de l'emploi, Paris, Armand Colin, 2003.

L. LIMA, « Le temps de la prime insertion professionnelle : un nouvel âge de la vie », dans A.A-M. Guillemard (dir), Où va la protection sociale?, Paris, PUF, 2008, pp. 49-67.

A. Poli, C. LEFRAnÇOIS et V. CARADEC, « Les discriminations liées à l'âge, entre exclusion et protection », Mouvements, le 23 septembre 2009.

A. Percheron, «Police et gestion des âges », dans A. Percheron et R. Remond (dir.), Age et politique, Paris, Economica, 1991, pp. 11-33. 